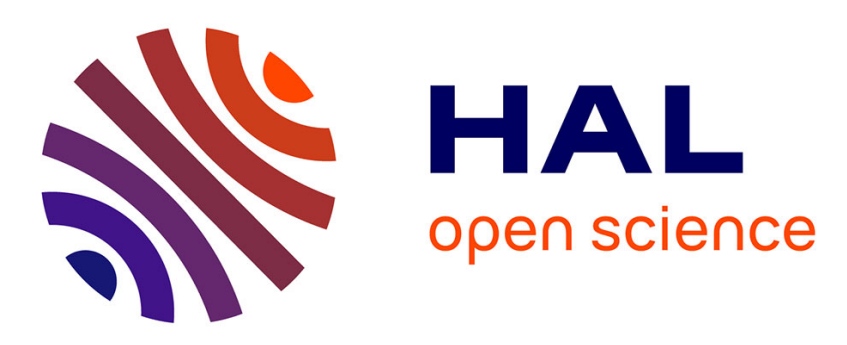

\title{
Synthesis of Homogeneous FePt Nanoparticles Using a Nitrile Ligand
}

\author{
Virginie Monnier, Michael Delalande, Pascale Bayle-Guillemaud, Yves
}

Samson, Peter Reiss

\section{To cite this version:}

Virginie Monnier, Michael Delalande, Pascale Bayle-Guillemaud, Yves Samson, Peter Reiss. Synthesis of Homogeneous FePt Nanoparticles Using a Nitrile Ligand. Small, 2008, 4 (8), pp.1139 - 1142. 10.1002/smll.200800217 . hal-01846293

\section{HAL Id: hal-01846293 \\ https://hal.science/hal-01846293}

Submitted on 23 Jul 2018

HAL is a multi-disciplinary open access archive for the deposit and dissemination of scientific research documents, whether they are published or not. The documents may come from teaching and research institutions in France or abroad, or from public or private research centers.
L'archive ouverte pluridisciplinaire HAL, est destinée au dépôt et à la diffusion de documents scientifiques de niveau recherche, publiés ou non, émanant des établissements d'enseignement et de recherche français ou étrangers, des laboratoires publics ou privés. 
FePt nanoparticles

\section{DOI: $10.1002 /$ smll.200800217}

\section{Synthesis of Homogeneous FePt Nanoparticles Using a Nitrile Ligand**}

\author{
Virginie Monnier, Michaël Delalande, Pascale Bayle- \\ Guillemaud, Yves Samson, and Peter Reiss*
}

Assemblies of monodisperse FePt nanoparticles (NPs) are promising candidates for next-generation data storage media. Recording densities beyond 1 Tbit inch $^{-2}$ can be envisioned ${ }^{[1]}$ due to the high uniaxial magnetocrystalline anisotropy of this alloy, which can reach $10^{7} \mathrm{~J} \mathrm{~m}^{-3}$ along the [001] direction of the face-centered tetragonal (fct) $L 1_{0}$ phase. ${ }^{[2]}$ Triggered by the seminal work of Sun and co-workers, ${ }^{[3]}$ the chemical synthesis of FePt NPs has been extensively studied in the last few years. ${ }^{[4]}$ Unfortunately, as-prepared NPs exhibit the chemically disordered face-centered cubic (fcc) structure. Therefore, their post-synthetic treatment by thermal annealing is necessary to form the desired ordered fct structure. This phase transition is only possible for $\mathrm{Fe}_{x} \mathrm{Pt}_{100-x} \mathrm{NPs}$ with $x$ in the range of $40-68 .{ }^{[5]}$ However, despite remarkable progress in the synthesis of size- and shape-controlled FePt NPs, ${ }^{[6,7,8 c]}$ a robust method for the preparation of particles exhibiting homogeneous composition is lacking. It has been shown that the initially reported synthesis ${ }^{[3]}$ as well as derived methods ${ }^{[6,8]}$ systematically lead to NPs with an Fe-depleted core and an Fe-rich shell. ${ }^{[9,10]}$ Such a core-shell structure is, of course, disadvantageous in view of the subsequent transformation to the $L 1_{0}$ phase. Based on theoretical calculations, it can be expected that a higher amount of thermal energy is required for the phase transition and that a lower degree of ordering per particle is obtained. ${ }^{[11]}$

The common scheme of the reported synthetic procedures, carried out in a high-boiling-point ether, is the reduction of platinum acetylacetonate, $\mathrm{Pt}(\mathrm{acac})_{2}$, accompanied by thermal decomposition of the iron precursor in the presence of a combination of oleic acid (OAc) and oleylamine (OAm)

[*] Dr. V. Monnier, Dr. P. Reiss

INAC (Institut Nanosciences et Cryogénie)/

SPrAM (UMR 5819)/LEMOH

CEA Grenoble

17 rue des Martyrs, 38054 Grenoble (France)

Fax: $(+33)$ 438-78-51-13

E-mail: peter.reiss@cea.fr

Dr. M. Delalande, Dr. P. Bayle-Guillemaud, Dr. Y. Samson

INAC/SP2M/NM and INAC/SP2M/LEMMA

CEA Grenoble

17 rue des Martyrs, 38054 Grenoble (France)

[**] This work was supported by the French National Research Agency (ANR, project CAMAIEU). Dr. J.-L. Rouvière is thanked for assistance with TEM.

․․․ Supporting Information is available on the WWW under http:// www.small-journal.com or from the author. stabilizers. In addition to the originally used iron pentacarbonyl $\left[\mathrm{Fe}(\mathrm{CO})_{5}\right]$, several ionic $\mathrm{Fe}$ precursors have been studied. ${ }^{[6 \mathrm{~b}-\mathrm{d}, 8]}$ However, in spite of these modifications NPs with uniform $\mathrm{Fe}$ distribution could not be obtained. We attribute the formation of NPs with an Fe-depleted core to the different reaction kinetics of the Fe and Pt precursors, which strongly depends on their interaction with the stabilizing ligands. In the reported reactions, OAc preferentially binds to $\mathrm{Fe}$ atoms, whereas OAm has a higher affinity for Pt atoms. ${ }^{[7 \mathrm{a}]}$

Herein, we describe a general strategy for the synthesis of FePt NPs with a large size range $(3-16 \mathrm{~nm})$, which show homogeneous radial composition and close to equimolar stoichiometry. With the goal to equilibrate the reactivity of the $\mathrm{Fe}$ and Pt precursors, we chose a nitrile surfactant, namely pentadecanenitrile (PN), which binds more strongly to $\mathrm{Pt}$ atoms than OAm. The use of this new surfactant has dramatic consequences on the size, size distribution, stoichiometry, and composition of the resulting FePt NPs.

FePt NPs were synthesized following the procedure reported by Sun et al., ${ }^{[3]}$ but replacing OAm with PN (see Experimental Section). A striking feature of the as-prepared NPs is revealed by transmission electron microscopy (TEM) analysis (Figure 1): a bimodal size distribution is obtained, independently of the [OAc]:[PN] ratio used. In the reaction leading to the NPs shown in Figure 1, application of $0.5 \mathrm{mmol}$ of OAc and $0.5 \mathrm{mmol}$ of PN yielded spherical FePt NPs 3.86 and $14.4 \mathrm{~nm}$ in diameter. Both populations showed narrow size distributions with a standard deviation of 9 and $7 \%$, respectively (Figure S1, Supporting Information). After separating the two size fractions by size-selective precipitation, ${ }^{[12]}$ the mass fraction of the smaller NPs was determined as $80 \%$. High-resolution TEM (HRTEM) studies (see insets in Figure 1) show that the $3.86-\mathrm{nm}$ NPs are essentially monocrystalline, while in the case of the 14.4-nm NPs, several orientations of the lattice planes are observed within one particle, which indicates polycrystallinity.

The mean size of each fraction of NPs can be controlled by the $[\mathrm{OAc}]:\left[\mathrm{Fe}(\mathrm{CO})_{5}\right]$ ratio, which indicates substantial participation of the $\mathrm{Fe}$ precursor in the nucleation process. As visible in Figure 2, for a given concentration of OAc the variation of the concentration of PN has little effect on the mean diameter of the NPs (smaller size fraction analyzed here). The newly developed synthetic method gives access to an unprecedented large range of diameters, from 3 to $6 \mathrm{~nm}$ for the smaller and from 12 to $16 \mathrm{~nm}$ for the bigger NPs (Figure S2). For both the smaller and the larger NPs obtained in one synthesis, the global composition was nearly equiatomic $\left(\mathrm{Fe}_{50} \mathrm{Pt}_{50}\right)$, as evidenced by energy-dispersive $\mathrm{X}$-ray (EDX) analysis (Figure S3). More information about the elemental distribution within individual particles was obtained by energy-filtering TEM (EFTEM), which enabled the recording of chemical maps (Figure 3 and Figure S4). For comparison, a sample synthesized using the standard procedure ${ }^{[3]}$ was also investigated. It clearly appears that the NPs synthesized with $\mathrm{OAm}$ as the Pt ligand present a core-shell structure, with an iron-depleted core (Figure 3a and d). In contrast, NPs synthesized with the PN stabilizer unambiguously exhibit a more homogeneous elemental distribution, both for bigger (Figure $3 b$ and e) and smaller NPs (Figure $3 c$ and f). 


\section{communications}
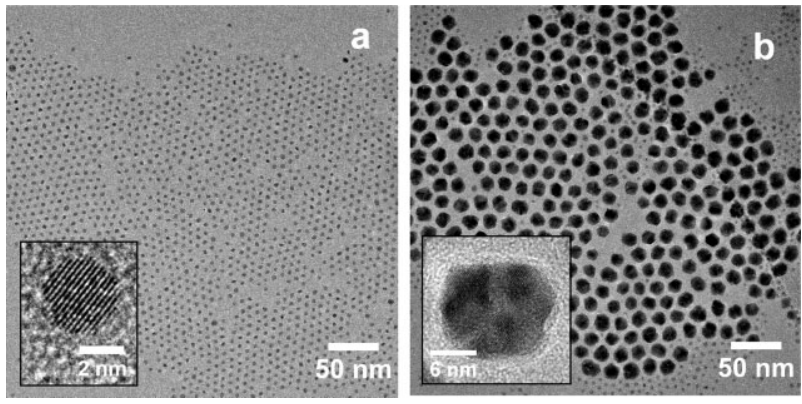

Figure 1. TEM images of as-synthesized FePt NPs. The use of OAc and PN as stabilizers results in a bimodal size distribution. a) Region with 3.86-nm NPs; b) zone containing a majority of 14.4-nm NPs (insets: HRTEM images).

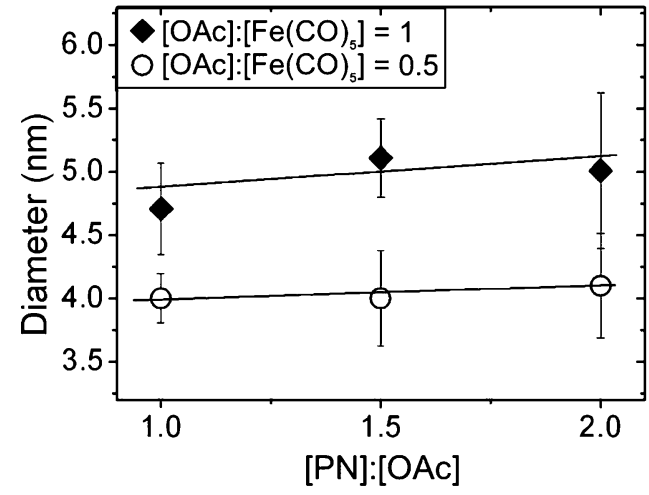

Figure 2. Mean size of the FePt NPs obtained (smaller size fraction) as a function of the $[\mathrm{PN}]:[\mathrm{OAc}]$ and $[\mathrm{OAc}]:\left[\mathrm{Fe}(\mathrm{CO})_{5}\right]$ ratios used. The error bars correspond to the size distributions determined with TEM.

As expected, the diffractogram of the 3.86-nm NPs (Figure 4a) exhibits broader diffraction peaks than that of the 14.4-nm NPs (Figure 4c). On the basis of Scherrer's formula, for the 3.86-nm NPs an average particle size of $3.4 \mathrm{~nm}$ was estimated
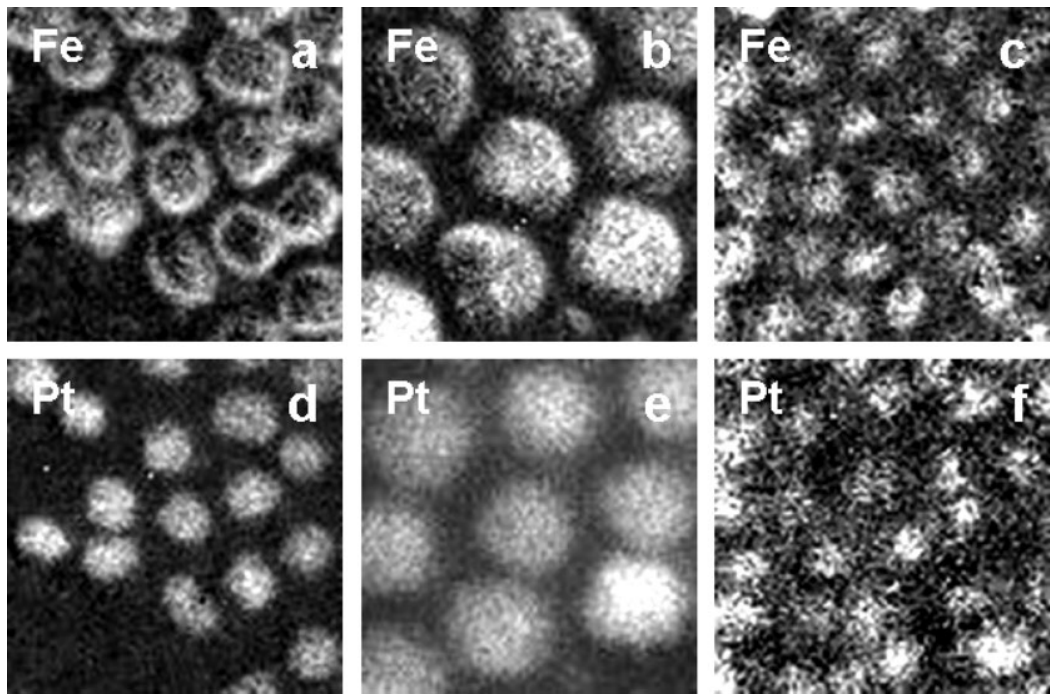

Figure 3. EFTEM chemical maps measured at the Fe and Pt thresholds: a),d) 7.0-nm FePt NPs synthesized with OAc/OAm; b),e) 14.4-nm NPs and c),f) 3.86-nm NPs produced with $\mathrm{OAC} / \mathrm{PN}$ stabilizing ligands. from the width of the X-ray diffraction (XRD) peaks, which is in good accordance with the diameter determined with TEM. On the other hand, the crystallite size obtained from X-ray analysis of the 14.4-nm NPs is only $6.1 \mathrm{~nm}$, which confirms their polycrystalline character. Both types of NPs crystallized in the fcc structure of the chemically disordered phase. The calculated lattice parameter $a$ was $3.8675 \AA$ for the smaller NPs and $3.8515 \AA$ for the larger ones. Using Végard's law, ${ }^{[13]}$ the corresponding core compositions were calculated as $\mathrm{Fe}_{42} \mathrm{Pt}_{58}$ and $\mathrm{Fe}_{46} \mathrm{Pt}_{54}$, respectively, which are very close to the global composition $\left(\mathrm{Fe}_{50} \mathrm{Pt}_{50}\right)$ measured by EDX analysis. In contrast, for the NPs produced with OAm as the Pt ligand, the core composition deduced from the lattice parameter $(a=3.884 \AA)$ is $\mathrm{Fe}_{30} \mathrm{Pt}_{70}$, which confirms the core-shell structure of these NPs.

Importantly, the homogeneous FePt NPs obtained with PN as the Pt ligand exhibit superior magnetic properties compared to NPs prepared with OAm. Figure 4e shows the magnetization as a function of temperature when applying a large field of $50 \mathrm{kOe}$. The Curie temperatures measured for the assynthesized NPs prepared with PN are significantly higher (383 K for 3.86-nm NPs; $>600 \mathrm{~K}$ for 14.4- and 16-nm NPs) than those of NPs prepared with OAm (260 K for 3.5-nm NPs), which agrees with the increased iron content of the magnetic cores of the NPs. Thermal annealing at $650{ }^{\circ} \mathrm{C}(1 \mathrm{~h}$, vacuum) induced the desired $\mathrm{fcc} \rightarrow \mathrm{fct}$ transformation for both size fractions of the NPs prepared with PN (Figure 4b,d). However, the narrowing of the peaks indicates coalescence of the NPs during the annealing process. The opened hysteresis loops observed at $T=300 \mathrm{~K}$ after annealing at $650^{\circ} \mathrm{C}$ (Figure S5) are consistent with the large anisotropy ferromagnetic $L 1_{0}$ phase.

Although small $(<4 \mathrm{~nm})$ FePt NPs synthesized with OAm can also be transferred into the $L 1_{0}$ phase by annealing at $650{ }^{\circ} \mathrm{C}$, this is not the case for bigger ones ( 5 to $7 \mathrm{~nm}$ ) obtained by using an excess of OAm and OAc. ${ }^{[6 a, 10]}$ Indeed, these NPs are still superparamagnetic at room temperature, and XRD analysis reveals that the $L 1_{2}$ structure $\left(\mathrm{FePt}_{3}\right)$ has been formed as a consequence of their overall Fe-depleted composition. On the contrary, when using PN as the Pt ligand, NPs of all size ranges (from 3 to $16 \mathrm{~nm}$ ) yield the $L 1_{0}$ phase after thermal annealing.

Stabilizing ligands play a crucial role in controlling the size, size dispersion, composition, and shape of chemically synthesized NPs. ${ }^{[14]}$ The key factor is the type and strength of interaction between the organic ligand and the metal atoms. The Fe-OAc interaction is rather strong, with energies approaching the values of covalent bonds. ${ }^{[15]} \mathrm{Pt}$ ions generally show a strong affinity towards ligands capable of establishing $\pi$ bonds, such as $\mathrm{CN}^{-}$or $\mathrm{NO}_{2}{ }^{-} \cdot{ }^{[16]}$ While the amine group can only act as a $\sigma$ donor, the nitrile function, isoelectronic with $\mathrm{CO}$, additionally acts as a $\pi$ acceptor in $\mathrm{d}_{\pi}-\pi^{*}$ bonds. ${ }^{[17]}$ We hypothesize that the formation of a stable Pt-nitrile complex results in comparable nucleation kinetics of the Pt and Fe precursors, which are at the 

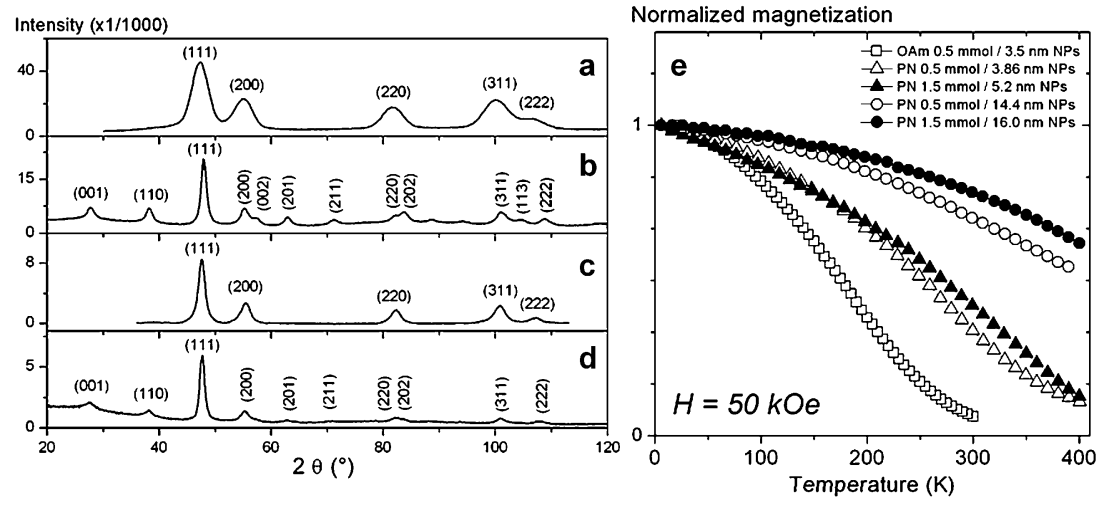

Figure 4. a-d) X-ray diffractograms of as-synthesized (a,c) and annealed (b,d) NPs (a,b: $3.86 \mathrm{~nm}, \mathrm{c}, \mathrm{d}: 14.4 \mathrm{~nm}$ ). e) Temperature-dependent normalized magnetization for various amounts of Pt ligands (OAc: $0.5 \mathrm{mmol}$ ).

origin of the observed homogenous composition of the NPs. As concerns the bimodal size distribution, our results clearly suggest that it is induced by the use of the PN ligand. A control experiment carried out under the same reaction conditions as for the syntheses presented in Figure 2 but using 2 mmol of PN as the only stabilizing ligand (i.e., without the use of OAc), yielded two populations consisting of 2.4- and 7-nm NPs. In contrast to $\mathrm{OAm}, \mathrm{PN}$ can act as a bridging ligand between $\mathrm{Pt}$ atoms and, furthermore, its shorter carbonaceous chain leads to an increase of the attractive interaction between particles, thus promoting their aggregation. ${ }^{[15,18]}$ Considering these elements, the formation of the large NPs could be attributed to an ordered, aggregative growth process, ${ }^{[19]}$ which competes with the classical growth by diffusion of the small particles. To get a better comprehension of the formation mechanism of the bimodal size distribution, aliquots were taken at different stages of the reaction and analyzed with TEM (Figure 5).

During the temperature increase from 100 to $287^{\circ} \mathrm{C}$, a notable color change of the reaction mixture was observed from pale yellow (P1) to dark yellow (P2), brown (P3), and then black (P4-P10). While P1 did not yield products analyzable with TEM, aliquot P2 was composed of small monodisperse NPs with a mean diameter of $2 \mathrm{~nm}$, which formed three-dimensional superstructures upon solvent evaporation (Figure 5b). Increasing the temperature to $200{ }^{\circ} \mathrm{C}(\mathrm{P} 3)$ resulted in the formation of bigger $(6-9 \mathrm{~nm})$ particles. Their interreticular distances determined from HRTEM images lie in the range of 2.214 $2.231 \AA$, that is, typical values of FePt alloy with close to equimolar composition. The small NPs of P2 were still visible; however, their supramolecular aggregation was no longer observed, which indicates that their concentration was much lower than at P2. At P4, two distinct size fractions of bigger $(12-13 \mathrm{~nm})$ and smaller NPs $(3.5 \mathrm{~nm})$ were visible, which at P8 partially auto-organized. Prolonging the heating for a further 16h (Figure 5f) reproducibly led to the nearly complete disappearance of the bigger NPs and resulted in a monodisperse population of 3.8-nm NPs $(\sigma=7.1 \%)$.

The observed behavior cannot be interpreted on the basis of the classical theory of Ostwald ripening, which implies the growth of larger particles at the expense of smaller ones,
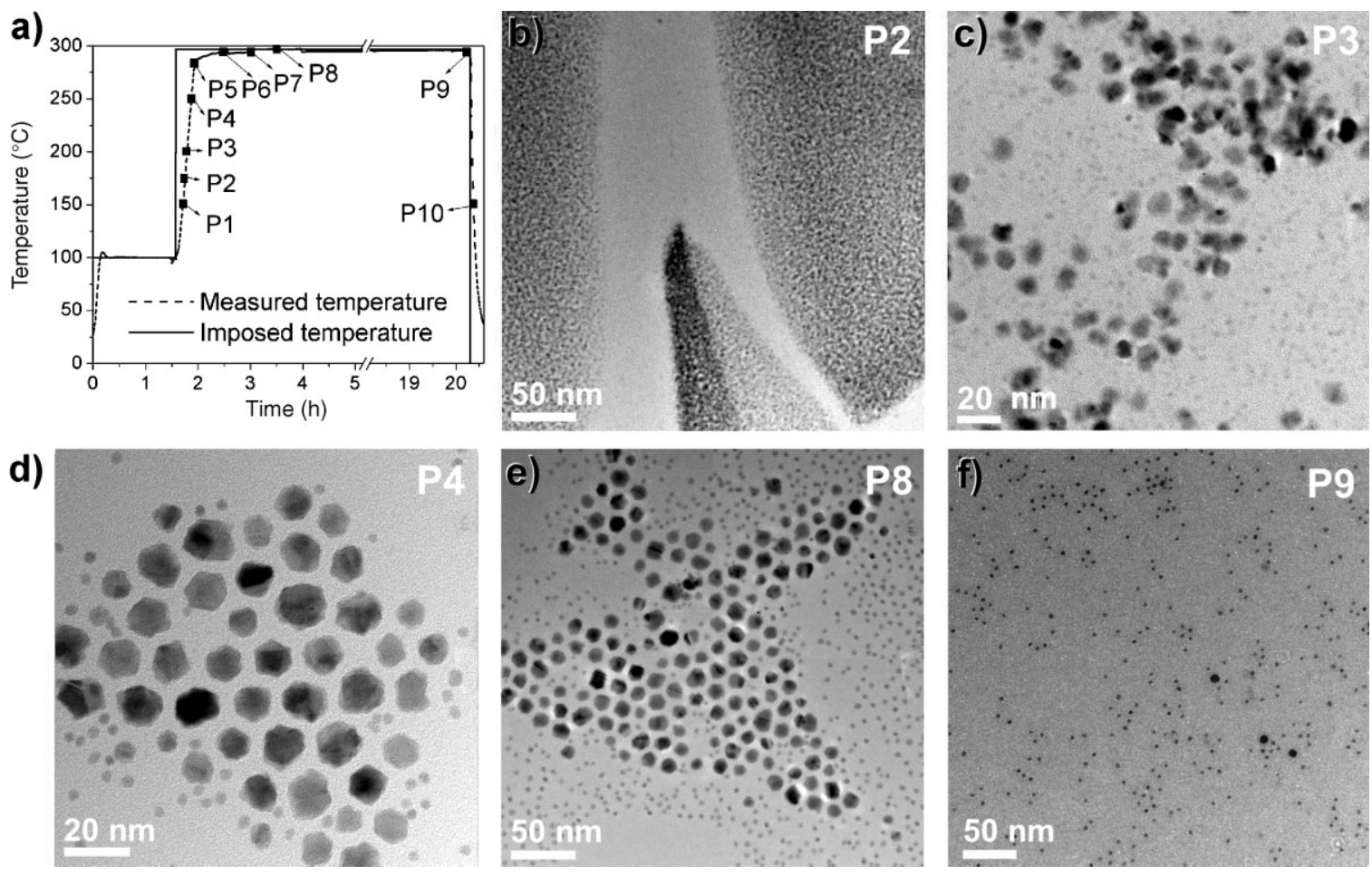

Figure 5. a) Evolution of the temperature of the reaction mixture during the synthesis of FePt NPs using $0.5 \mathrm{mmol}$ of $\mathrm{OAc}$ and $0.5 \mathrm{mmol}$ of PN. Aliquots taken at different stages of the reaction are indicated by P1 to P10. b-f) TEM images of selected aliquots. 
driven by a reduction of the total surface free energy. ${ }^{[20]}$ Prominent examples of the observed reverse ripening process, termed digestive ripening, are gold and silver NPs. ${ }^{[21]}$ Lee and co-workers recently proposed a theoretical model for digestive ripening, assuming charged particles. ${ }^{[22]}$ Our system follows intriguingly well the predicted evolution of size distribution, namely 1) formation of an initial bidisperse state consisting of distinct populations of small and large NPs, followed by 2) decay of the larger particles with time to finally yield a monodisperse population of small particles. However, further studies are necessary to unravel the chemical reactions involved in the formation of FePt NPs and to correlate theory and experiment in more detail.

In conclusion, we have demonstrated that Pt-ligand interaction plays a key role in the synthesis of FePt NPs. The use of PN gives access to a large range of NP diameters (3-16 nm) within two distinct size distributions. Importantly, both size fractions comprise monodisperse NPs of homogeneous radial composition and close to equimolar stoichiometry. It is expected that these homogeneous NPs will provide significant advantages over particles of core-shell type in their subsequent transformation to the $L 1_{0}$ phase. Using, for example, the recently developed salt-annealing technique, ${ }^{[23]}$ the presented synthesis method paves the way for obtaining a large size range of fully ordered fct FePt NPs with a high potential for application in magnetic data storage media.

\section{Experimental Section}

A stirred mixture of $\mathrm{Pt}(\mathrm{acac})_{2}$ (98\%; $\left.197 \mathrm{mg}, 0.5 \mathrm{mmol}\right)$ and 1,2hexadecanediol ( $90 \%$; $390 \mathrm{mg}, 1.5 \mathrm{mmol}$ ) in dioctyl ether $(99 \%$; $17 \mathrm{~mL}, 13.7 \mathrm{~g}$ ) was heated under argon at $100^{\circ} \mathrm{C}$ for $1 \mathrm{~h}$, which resulted in a light-yellow transparent solution. A mixture of $\mathrm{Fe}(\mathrm{CO})_{5}(99.99 \% ; 196 \mathrm{mg}, 1 \mathrm{mmol})$ and designated amounts (e.g., $0.5 \mathrm{mmol}$ each) of OAc (99\%) and PN (97\%) in dioctyl ether ( $3 \mathrm{~mL}$, $2.418 \mathrm{~g}$ ) was injected into the Pt-containing solution and the temperature was raised to $287^{\circ} \mathrm{C}$ at a rate of $10^{\circ} \mathrm{C} \mathrm{min}^{-1}$. Aliquots $(0.5 \mathrm{~mL})$ of the reaction mixture were taken at different time intervals and diluted with toluene $(1 \mathrm{~mL})$, followed by precipitation with ethanol and dispersion in fresh toluene. After $30 \mathrm{~min}$ of reflux, the reaction mixture was cooled to room temperature. Purification was carried out by adding anhydrous ethanol $(80 \mathrm{~mL})$ to the reaction mixture, centrifugation, and redispersion of the NPs in toluene $(20 \mathrm{~mL})$.

\section{Keywords:}

alloys · crystal growth · ligand design - magnetic properties · nanoparticles

[1] H. Kodama, S. Momose, T. Sugimoto, T. Uzumaki, A. Tanaka, IEEE Trans. Magn. 2005, 41, 665-669.

[2] D. Weller, M. F. Doerner, Ann. Rev. Mater. Sci. 2000, 30, 611-644.

[3] S. H. Sun, C. B. Murray, D. Weller, L. Folks, A. Moser, Science 2000, 287, 1989-1992.

[4] S. H. Sun, Adv. Mater. 2006, 18, 393-403.
[5] C. B. Rong, Y. Li, J. P. Liu, J. Appl. Phys. 2007, 101, 09. K505.

[6] a) M. Chen, J. P. Liu, S. H. Sun, J. Am. Chem. Soc. 2004, 126, 83948395 ; b) V. Nandwana, K. E. Elkins, N. Poudyal, G. S. Chaubey, K. Yano, J. P. Liu, J. Phys. Chem. C 2007, 111, 4185-4189; c) S. H. Sun, S. Anders, T. Thomson, J. E. E. Baglin, M. F. Toney, H. F. Hamann, C. B. Murray, B. D. Terris, J. Phys. Chem. B 2003, 107, 5419-5425; d) K. E. Elkins, T. S. Vedantam, J. P. Liu, H. Zeng, S. H. Sun, Y. Ding, Z. L. Wang, Nano Lett. 2003, 3, 1647-1649.

[7] a) M. Chen, J. Kim, J. P. Liu, H. Y. Fan, S. H. Sun, J. Am. Chem. Soc. 2006, 128, 7132-7133; b) C. Wang, Y. L. Hou, J. M. Kim, S. H. Sun, Angew. Chem. 2007, 119, 6449-6451; Angew. Chem. Int. Ed. 2007, 46, 6333-6335; c) M. Chen, T. Pica, Y. B. Jiang, P. Li, K. Yano, J. P. Liu, A. K. Datye, H. Y. Fan, J. Am. Chem. Soc. 2007, 129, 63486349.

[8] a) L. E. M. Howard, H. L. Nguyen, S. R. Giblin, B. K. Tanner, I. Terry, A. K. Hughes, J. S. O. Evans, J. Am. Chem. Soc. 2005, 127, 1014010141; b) H. L. Nguyen, L. E. M. Howard, S. R. Giblin, B. K. Tanner, I. Terry, A. K. Hughes, I. M. Ross, A. Serres, H. Bürckstümmer, J. S. O. Evans, J. Mater. Chem. 2005, 15, 5136-5143; c) H. L. Nguyen, L. E. M. Howard, G. W. Stinton, S. R. Giblin, B. K. Tanner, I. Terry, A. K. Hughes, I. M. Ross, A. Serres, J. S. O. Evans, Chem. Mater. 2006, 18, 6414-6424.

[9] S. Saita, S. Maenosono, Chem. Mater. 2005, 17, 6624-6634.

[10] M. Delalande, P. R. Marcoux, P. Reiss, Y. Samson, J. Mater. Chem. 2007, 17, 1579-1588.

[11] H. Bernas, J. P. Attane, K. H. Heinig, D. Halley, D. Ravelosona, A. Marty, P. Auric, C. Chappert, Y. Samson, Phys. Rev. Lett. 2003, 91, 077203.

[12] O. Perales-Perez, H. Sasaki, A. Kasuya, B. Jeyadevan, K. Tohji, T. Hihara, K. Sumiyama, J. Appl. Phys. 2002, 91, 6958-6960.

[13] A. Bonakdarpour, J. Wenzel, D. A. Stevens, S. Sheng, T. L. Monchesky, R. Lobel, R. T. Atanasoski, A. K. Schmoeckel, G. D. Vernstrom, M. K. Debe, J. R. Dahn, J. Electrochem. Soc. 2005, 152, A61-A72.

[14] a) E. V. Shevchenko, D. V. Talapin, H. Schnablegger, A. Kornowski, O. Festin, P. Svedlindh, M. Haase, H. Weller, J. Am. Chem. Soc. 2003, 125, 9090-9101; b) S. Kumar, T. Nann, Small 2006, 2, 316329; c) P. D. Cozzoli, T. Pellegrino, L. Manna, Chem. Soc. Rev. 2006, 35, 1195-1208; d) C. Burda, X. B. Chen, R. Narayanan, M. A. ElSayed, Chem. Rev. 2005, 105, 1025-1102.

[15] A. C. S. Samia, J. A. Schlueter, J. S. Jiang, S. D. Bader, C. J. Qin, X. M. Lin, Chem. Mater. 2006, 18, 5203-5212.

[16] F. A. Cotton, G. Wilkinson, in Advanced Inorganic Chemistry, 5th ed., Wiley-VCH, Weinheim 1988, p. 918.

[17] J. E. Huheey, E. A. Keiter, R. L. Keiter, Chimie Inorganique, De Boeck \& Larcier, Paris 1996, p. 421.

[18] J. J. Cerdà, T. Sintes, C. M. Sorensen, A. Chakrabarti, Phys. Rev. E 2004, 70, 0114051-0114059.

[19] M. Ocana, R. Rodriguez-Clemente, C. J. Serna, Adv. Mater. 1995, 7, 212-216.

[20] I. M. Lifshitz, V. V. Slyozov, J. Phys. Chem. Solids 1961, 19, 35-50.

[21] S. Stoeva, K. J. Klabunde, C. M. Sorensen, I. Dragieva, J. Am. Chem. Soc. 2002, 124, 2305-2311.

[22] D.-K. Lee, S.-I. Park, J. K. Lee, N.-M. Hwang, Acta Mater. 2007, 55, 5281-5288.

[23] a) K. Elkins, D. Li, N. Poudyal, V. Nandwana, Z. Q. Jin, K. H. Chen, J. P. Liu, J. Phys. D. Appl. Phys. 2005, 38, 2306-2309; b) C. B. Rong, D. R. Li, V. Nandwana, N. Poudyal, Y. Ding, Z. L. Wang, H. Zeng, J. P. Liu, Adv. Mater. 2006, 18, 2984-2988.

Received: February 10, 2008 Revised: April 23, 2008 Published online: July 11, 2008 